Gottieb B. y cols.

Rev. Chil, Pedlatr. 66 (6); 304-308, 1995

\title{
Control de la giardiadis en niños de jardines infantiles
}

\author{
Beatriz Gottlieb B.' ; Isabel Noemí H. '; Hernán Reyes M.'1; \\ Víctor Muñoz F. ${ }^{2}$, Carolina Valencia O.', Vicky Roizen G. ${ }^{3}$
}

\section{Control of Giardia lamblia infection among kindergarten children}

Three different scredules of treatment for Giardia lamblio infection were evaluated in 416 children from poor neighbouthoods kindergarten schools by parasitologicol excmiration of fecal somples. In the firsi kindergarten lgroup I|. children were only given oral tinidazol $50 \mathrm{mg} \cdot \mathrm{kg}$ of body weight for three times, af diagnosis ond later at three monlhs intervals. In the second one [group Il] the same trealmenl schedve was given logether with health education on preventive measures on each one of the three opcrtunities. Children attending to the third kindergarren lgroup ill received pharmacological treatment only wice, al diagnosis and atter six months, but health education wos given thrice. also at three month intervals from diagnosis. The frequency of infection lowered significantly from $42.9 \% 10$ $17.5 \%$ al the end of the school year $\{<<0.051$ in group it potients, but only from $36.3 \%$ to $25.4 \%$ in group 1 and from $50.5 \% 1021.5 \%$ in group \#ll. Thus, giardicsis seems to be best controlled by boh periodicol ireatment ond health educatlon of infected critidren.

(Koy words: Giordia lombira, school chilcren, treament.|

Los parásitos intestinales pueden causar trastornos en el crecimiento y desarrollo de los niños menores ${ }^{1-6}$. En jardines infantiles de Santiago, la frecuencia de infección de estos niños con dichos agentes es muy alta y la mayor proporción corresponde a Giardia lambia (más de $40 \%)^{7 \cdot 12}$. Este hecho, también descrito en países con más desarrollo relativo, como los Estados Unidos de Norteamérica ${ }^{2426}$, configura a esta parasitosis como un importante problema de salud pediátrica. Como, además, las tasas de esta infección son mayores en jardines infantiles de los sectores más pobres de la ciudad ${ }^{13-17}$, e1 daño social que ella provoca se acentúa entre los babitantes más expuestos a riesgo y más vulnerables a sus efectos patogénicos. En busca de soluciones al problema, se han ensayado el tratamiento de los individuos infectados (con resultados poco duraderos por rápida reinfección

1. Unidad de Parasitología, Campus Oriente, Departanento de Parasitología. Facultad de Medicina. Universidad de Chile.

2. Tecnólogo Médiço. Unidad de Parasitología, Campus Oriente, Departamento de Parasitologia, Facultad de Medicina, Universidad de Chile.

3. Alumna. Facultad de Medicina. Universidad de Chile. de ellos) y el de los infectados y su familia (lo que ha permitido controlar la infección sólo a mediano plazo, pero con tasas de infección iguales o superiores a las iniciales al cabo de seis meses ${ }^{18}, 22$ ). Si se agrega educacion sanitaria, el porcentaje de infectados es menor y el plazo de reinfección mayor, pero aun así, las cifras siguen siendo altas, entre 30 y $40 \%$.

Dado el favorable efecto clínico observado en los niños infectados tras la erradicación del parásito, que se expresa en recuperación del peso y aumento de la talla, es deseable impedir, o al menos retardar en todo lo posible, la infección reiterada por $G$. lamblia en los nenores que habitan en sectores de extrema pobreza. Con este propósito se aplić́ un programa de educación sanitaria y tratamiento de los niños infectados, que fue repetido periódicamente, en tres jardines infantiles de baja condición socioeconómica de Santiago, Chile. Para efectos de comparacion, en una de estas instituciones se aplicó el programa completo y en las otras dos, en forma parcial, como se indica más adelante. Las respectivas comunidades fueron debidamente informadas de las características y objetivos del programa que se aplicaría en cada jardín, insistiéndose en que -en todo caso- los niños 
infectados serían debidamente tratados y controlados al conocerse los resultados.

\section{Material y Método}

Se seleccionaron al azar tres jardines infantiles de sectores de extrema pobreza de Santiago, que fueron denominados jardines I, II y UI. La experiencia se realizó en cuatro etapas sucesivas: una inicial de diagnóstico. dos de control y una final de evaluacion, separadas por intervalos de tres meses. El programa se inicio con un curso de educación sanitaria sobre enteroparasitosis impartido por los investigadores a las educadoras de párvulos y auxiliares de los jardines II y III en el mes de enero. Su propósito fue preparar monitoras que hiciesen llegar esta información a la comunidad escolar. En él se diseñaron las actividades de educación sanitaria de todo el año. Según el caso, se planificaron charlas, discusiones de grupo. desartollo de unidades educativas. En la programación y desarrollo de estas actividades participaron también los investigadores. A continuación y al comienzo del ano escolar, en el mes de marzo, se encuestaron, mediante tres muestras para examen coproparasitológico seriado, los niños de los tres jardines, con el objeto de determinar la frecuencia de infección por prolozoos y helmintos intestinales. En la recolección y el exanten de las muestras fecales se utilizó el método de Burrows o PAFS ${ }^{19}$, examinando -en total- las deposiciones de 416 niños.

En los uiños del jardín I sólo se efectuó tratamiento de los infectados. Este se realizo con tinidazol, en dosis oral única de $50 \mathrm{mg} \cdot \mathrm{kg}$ de peso después del examen en que se hizo el primer diagnóstico y después del primer $y$ segundo controles realizados tres y seis meses más tarde. A los niños del jardín II se les administro igual tratamiento $\mathrm{y}$, además, se les dio educación sanitaria después del examen diagnóstico inicial, como también en el primero y el segundo controles. En el jardín III se hizo el mismo manejo que en el caso anterior, pero en el primer control, tres meses después del examen inicial, sólo se hizo educación sanitaria

Se practicaron, en total, tres controles, tres, seis y nueve meses después de la encuesta inicial (junio, septiembre y diciembre), mediante exámenes coproparasitarios senados de muestras obtenidas de los nifios de cada jardín. empleando el mismo método que en el examen diagnóstico inicial. Después de efectuado el último control se realizó la educación sanitaria a los integrantes del jardín I, en quienes no se habla hecho por programa.

\section{Resultados}

En $51,7 \%$ de los 416 niños encuestados mediante el examen inicial se encontraron infecciones por protozoos y helmintos intestinales sin diferencias significativas entre los asistentes a los jardines I, II y III, en que las proporciones de infectados fueron, respectivamente, $48,1 \%$ $(90 / 187), 53 \%(70 / 132)$ y $56,7 \%(55 / 97)$. Entre las especies de protozoos y helmintos identificados en el examen inicial, $G$, lamblia fue el más frecuentemente detectado, en $42,1 \%$ de los niños.

\section{Tabla 1}

Protozoos y helmintos intestinales detectados en niños de tres jardines infantiles de sectores de extrema pobreza, Santiago. Resultados del examen coproparasitológico inicial

\begin{tabular}{|c|c|c|c|c|c|c|c|c|}
\hline \multirow{3}{*}{ Parásitos } & \multicolumn{8}{|c|}{ Jardín Jnfantil } \\
\hline & \multicolumn{2}{|c|}{$\begin{array}{c}\mathrm{I} \\
\text { (n: 187) }\end{array}$} & \multicolumn{2}{|c|}{$\begin{array}{c}\text { II } \\
\text { (n: 132) }\end{array}$} & \multicolumn{2}{|c|}{ (III } & \multicolumn{2}{|c|}{$\begin{array}{c}\text { Total } \\
\text { (n: 416) }\end{array}$} \\
\hline & $\mathbf{n}$ & $\%$ & $\mathbf{n}$ & $\%$ & $\mathbf{n}$ & $\%$ & $\mathbf{n}$ & $\%$ \\
\hline $\begin{array}{l}\text { Giardia } \\
\text { Lambdia }\end{array}$ & 68 & 36,3 & 58 & 43,9 & 49 & 50,5 & 175 & 42,1 \\
\hline $\begin{array}{l}\text { Endolimax } \\
\text { nana }\end{array}$ & 13 & 7,0 & 8 & 6,1 & 13 & 13,4 & 34 & 8,2 \\
\hline $\begin{array}{l}\text { Entamoeba } \\
\text { coli }\end{array}$ & 13 & 7.0 & 17 & 12,9 & 12 & 12.14 & 32 & 7,7 \\
\hline $\begin{array}{l}\text { Hymenolepis } \\
\text { noma }\end{array}$ & 2 & 1,1 & 4 & 3,0 & 1 & $\mathbf{1}, 0$ & 7 & 1,7 \\
\hline $\begin{array}{l}\text { Entamoeba } \\
\text { histolytica }\end{array}$ & 2 & 1,1 & - & - & 1 & 1.0 & 3 & 0.7 \\
\hline $\begin{array}{l}\text { Chilomastix } \\
\text { maslini }\end{array}$ & - & - & 1 & 0.8 & 2 & 2,1 & 3 & 0,7 \\
\hline Taenia & & & & & & & & \\
\hline$s p$ & - & - & - & - & 1 & 1,0 & 1 & 0,2 \\
\hline
\end{tabular}


Luego de aplicar los tres diferentes esquemas de procedimiento y control, la evolución de los porcentajes de infección por $G$. lamblia fue, para cada caso, la indicada en la tabla 2 . Si bien en los tres jardines se obtuvo porcentajes finales de infección menores que los iniciales, sin embargo la magnitud del descenso fue diferente en cada uno. De acuerdo a la significación estadística por diferencia de proporciones, en el jardín infantil Il, donde la educación sobre prevención y tratamiento fue reiterada a lo largo de todo el programa, se logró el mejor control de la giardiasis, con tasas de infección inicial de $43,9 \%$ y final de $17.5 \%(p<0,05)$. El esquema aplicado en el jardín IП permitió llegar a una cifra de infección similar a la del jardín $I$, a pesar que inicialmente, por azar, en este último se había encontrado menor frecuencia de la parasitosis. En el jardín I, aunque al comienzo se redujo la frecuencia de infección por $G$. lamblia de $36,3 \%$ a $26 \%$ ( $p<0,05$ ), los tratamientos medicamentosos sucesivos ulteriores sólo consiguieron mantenerla estable, sin aminorarla.

\section{Comentario}

G. lamblia es el parásito intestinal más frecuente en los niños, especialmente en la edad en que ellos asisten a jardines infantiles ${ }^{13-18}$.

Los niños que ingresan a estos establecimientos presentan, al inicio del año, tasas de infección similares al promedio nacional, pero en los meses siguientes éstas aumentan progre- sivamente hasta alcanzar más de 60 o $70 \%$, lo que pudiese favorecer el traspaso de la parasitosis de infectados a sanos -mientras permanecen en el jardín-o hacia y desde el personal que les atiende, a lo que contribuirian la estrecha y prolongada convivencia entre las personas que ocupan estos recintos, y posibles fallas en las medidas higiénicas para prevenir estas infecciones. Este factor debe ser tomado en cuenta, como también que la adopción de las precauciones del caso debiera formar parte de las normas de atención y manejo de codos los jardines infantiles.

A pesar de estos altos índices, no siempre los exámenes coproparasitológicos seriados detectan todos los casos de infección por el parásito. Es así como en 250 niños de la ciudad de Santiago con examen de deposiciones negativo, se detectó $G$. lamblia por otros métodos (biopsia intestinal, análisis de jugo intestinal ${ }^{20}$, lo que implica que al evaluar niños en jardines infantiles empleando sólo el examen de las deposiciones, probablemente se está detectando menos infectados de los que existen. Por otra parte, la infección es de alta transmisibilidad (60\% de los familiares que viven bajo el mismo techo de un caso índice también están infectados) ${ }^{12}$ y casi $50 \%$ de los niños infectados son asintomáticos ${ }^{20}$, a pesar de lo cual aún hay controversia sobre la conducta que debe seguirse frente a esios casos ${ }^{26-30}$.

Los resultados obtenidos en estudios previos han sido alentadores, pero no suficientes. Las acciones esporádicas de tratamiento o educa-

\section{Tabla 2}

Giardiasis en niños de tres jardines infantiles de sectores de extrema pobreza, Santiago. Evolución de la infección según esquema aplicado

\begin{tabular}{lcccccc}
\hline & $\begin{array}{c}\text { I } \\
\text { Positivos/ } \\
\text { examinados }\end{array}$ & $\%$ & $\begin{array}{c}\text { II } \\
\text { Positivos/ } \\
\text { examinados }\end{array}$ & $\%$ & $\begin{array}{c}\text { UI } \\
\text { Positivos/ } \\
\text { examinados }\end{array}$ & \% \\
\hline $\begin{array}{l}\text { Exámen } \\
\text { inicial }\end{array}$ & $68 / 187$ & 36,3 & $58 / 132$ & 43,9 & $49 / 97$ & 50,5 \\
$\begin{array}{l}\text { Control } 1 \\
(3 \text { meses })\end{array}$ & $44 / 169$ & 26,0 & $15 / 130$ & 12,1 & $14 / 90$ & 15,5 \\
$\begin{array}{l}\text { Control 2 } \\
(6 \text { meses) }\end{array}$ & $43 / 185$ & 23,1 & $14 / 124$ & 11,2 & $31 / 88$ & 35,2 \\
$\begin{array}{l}\text { Control 3 } \\
(9 \text { meses) }\end{array}$ & $46 / 181$ & 25,4 & $21 / 120$ & 17,5 & $19 / 87$ & 21,8 \\
\hline
\end{tabular}


ción sanitaria no resuelven satisfactoriamente el problema, por muy amplias o sistemáticas que hayan sido, pues al cabo de pocos meses la situación ha recaído a su magnitud inicial, si bien programas de tratamiento y educación sanitaria repetidos dos veces con lapso de ocho meses han permitido lograr descender en forma significativa las cifras iniciales, aunque sin 10 grar aún un buen control de la reinfección ${ }^{18.22}$.

En la experiencia que se comenta destaca claramente la mejor evolución final observada en el jardín II, donde además de tratamiento reiterado se aplicó sistemáticamente la educación pertinente. Además, aun cuando las tasas iniciales de infección por $G$. lambdia en los jardines I y III era, por azar, mayor en el último, al final del estudio fueron similares. Considerando que en el jardín I s6lo se aplicó tratamiento de la giardiasis en tres oportunidades, sin hacer educación, mientras en el jardín III se hicieron dos tratamientos (cada seis meses), pero con educación sanitaria, este hecho sugiere que el tratamiento con educaciớn es económicamente más ventajoso.

\section{Resumen}

Se evaluaron tres esquemas de tratamiento y control de las giardiasis. Para ello se estudiaron, mediante examen coproparasitológico (PAFS), muestras seriadas de deposiciones de 416 niños de tres jardines infantiles de extrema pobreza, encontrándose una tasa global de $42,1 \%$ de infección por Giandia lamblia. Los niños del primer jardín infantil $(n=187)$ recibieron solo tinidazol $50 \mathrm{mg}$ * $\mathrm{kg}$ cada tres meses en tres oportunidades. Los del segundo jardín ( $n=132)$ recibieron el medicamento y educación sanitaria en las mismas tres oportunidades. A los de la tercera unidad ( $n=97$ ) se les administró el medicamento sólo dos veces a intervalos de seis meses, pero se les hizo educación sanitaria trimestral en tres ocasiones. Al cabo de nueve meses de seguimiento, la tasa de infecciones por Giardia lamblia disminuyó de $36,3 \%$ a $25,4 \%$ en el primer caso, de $43,9 \%$ a $17,5 \%$ en el segundo $y$ de $50,5 \%$ a $21,8 \%$ en el tercero, sugiriendo un importante efecto favorable $(p<0,05)$ de las medidas de educacion sanitaria y prevención sobre la tasa de reinfección y la latencia de ésta en jardines infantiles de extrema pobreza.
(Palabras clave: Giardia Lamblia, tratamiento, prevención.)

\section{Referencias}

I. Farthing M, Masa L, Urrutia J, Kronnal R: Natural history of Giardia infection of infants and children in rural Guaternala and its impact on physical growth. Am J Clin Nutrition 1986; 43: 395-405.

2. Hjels $K$, Paerregaard, Krasinikoff $P$ : Giardiasis causing chronic diarthoea in suburban Copenhagen: incidence, physical prowth, clinical symptoms and small intestinal abnormality. Acta Paediatr Scand 1992; $81: 881-886$.

3. Chunge $R$, Karumba $P$, Kaleli $N$, et at:. Prevalence and frequency of Giardia lamblia in children aged 0 to 60 months with and diarrhoea. East Afr Med J 1992: 69: 311-313.

4. Hjelt $K$, Paerregaard A, Kravilnikoff P: Giardiasis: Hamatological status and the absorption of vitamin $\mathrm{B} 12$ and folic acid. Acta Paediatr Scand 1992;81: 29-34.

5. Kateralis $P_{1}$ Farthing $M$ : Diarthoea and malabsorption in giardiasis: a multifactorial process? Gut 1992; 33 : 295-297.

6. Farthing M: Diarrboeal disease: current concepts and future challenges. Pathogenesis of giardiasis. Trans $\mathbf{R}$ Soc Trop Med Hyg 1993; 87: 17-2I.

7. Ramirez $R$, Schenone $H$, Galdames $M$, et al.: Frecuencia en Chile de las infecciones por protozoos y helmintos intestinales (1962 - 1972). Bol Chil Parasitol 1972; 27: 116-118

8. Noemí 1 . Reyes H. Mauro G: Enleroparasitosis en la población infantil del Area de Salud Oriente de Santiago. Rev Chil Pediatr 1974; 45: 454-457.

9. Frenzel A, Torres P. Guerrero $S$, Gesche $W$, Montefuso A, Marin F: Parasitosis intestinal en lactantes y su relación con la infección de sus manipuladoras de alimentos $y$ el saneamiento ambiental. Rev Med Chile 1979: 107: 343-351.

10. Schenone $H$, Rojas A, Galdames $M$, Villarroel F: Aspectos epidetmológicos de las infecciones humanas por protozoos y helmintos intestinales en Chile (1970-1980). Bol Chil Parasitol 1981; 36: 44-48.

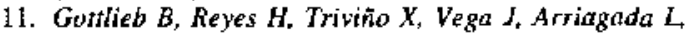
Hernandez $J$ : Enteroparasitosis en niños de jardines infantiles. Estudio comparativo según njvel socioeconúmico. Rev Med Chile 1983; 111: 1035-1038.

12. Gottlieb B, Tapia R, Reyes H: Giardiasis familiar. Estudio clínico y epidemiológico a partir de casos indices. Rev Med Chile 1982: 110: 224-227.

13. Schenone $H$, Villarroel $F$ : Algunos aspectos epidemiológicos de las enteroparasitosis en nißoos de hogares de menores de Santiago de Chile. Bol Chil Parasitol $1978 ; 33: 78-82$.

14. Noemt I, Reyes $H$, Mauro $G$ : Infecciones por enteroparásitos en niños de sala cuna y jardín infantil de dos hospitales de Santiago. Rev Chil Pediatr 1975; 46:50.53.

15. Schenone $H$, Salyedra $T$, Galdames $M$, Inzunza $E$, Jimenez $M$. Romero $E$ : Epidemia de giardiasis en un 
jardín infantil y el uso de nimorazol en su control. Bol Chil Parasitol 1976; 31: 12-15.

16. Noemi I: lnfección por enteroparásitos en jardines infantiles y salas cunas de Santiago. Vigilancia de Enfermedades Transmisibles y Zoonosis (Chile) 1978; 5: 7-9.

17. Noemí 1 , Reyes H, Hemandez J: Enteroparasitosis en niros de salas cunas y jardines infantiles de Sanuago. Rev Med Chile 1983; 111: 802-807

18. Gotlieb 8 , Agutlera $X$, Reyes $H$, et al.: Enteropasitosis en jardines infantiles. Evahuación de algunas normas de control. Parasitologia al Dia 1984; 8: 36 42 .

19. Burrows $R$ : A new fixative and technics for the diagnosis of intestinal parasites. Am J Clin Pathol 1967: 48:342-346.

20. Guiraides E. Venegas G. Gutierrez $C$, Mauro $G$, La Torre J. Noemi I: Estodio comparativo de tres métodos en el diagnóstico de giardiasis. Rev Med Chile $1982 ; 110 ; 21-23$.

21. Steketee $R$, Reid $S$, Cheng $T$, et al: Recurrent outbreaks of Giardiasis in a child day care center, Winconsin. Am J Public Health 1989; 79: 485-490.

22. Gildman $R$, Marguis $G$, Miranda $E$, Vestegui $M$. Mortínez H: Rapid seinfection by Giardia lamblia after treatment in a hyperendemic third world comnutity. Lancet 1988; 13: 343-245.
23. Addiss $D$, Juranek $D$, Spencer $H$ : Treatment of children with asyntomatic and nondiartheal Giardia infection. Pediatric Infect Dis J 1991; 10:843-846.

24. Overturf $G$ : Editorial Response: Endemic Giardiasis in the United States-Role of the Day-Care Center. Clin Infect Dis 1994; 18: 764-765.

25. Addiss D, Davis J, Roberts J, Mast E: Epidemiology of giardiasis in Wisconsin: increasing incidence of reported cases and unexplained seasonal trends. Am J Trop Med Hyg 1992; 47: 13.

26. Rauch A. Barlett A. Pickering L: Longitudinal study of Giardia lamblia infection in a day-care center population. Pediatr Infect Dis J 1990: 9: 186-189.

27. Nigam $P$, Kapoor $X$, Kumar A. Sarkari $N$. Gupta A: Clinical profile of giardiasis and comparison if this therapenthic response to metronidazole and tinidazole. J Assoc Physicians India I991; 39: 613-615.

28. Addis $D$. Juranek $D$, Spencer $H$. Treatment of children with asyntomatic and nondiarrheal Giardia infection. Pediatr Infect Dis J 1991: 10: 843-846.

29. Fraser D: Epidemiology of Giardia lamblia and Coptosporidium infection in childhood. lsr J Med Sci 1994: 30:356-361.

30. Rousham E: An increase in Giardia duodenalis infection among children reciving periodic antihelminthic treatment in BangJadesh. J Trop Pediatr 1994; 40: $329-333$.

\section{AVISO A LOS AUTORES}

Por acuerdo del Comité Editorial, la Revista Chilena de Pediatría devolverá sin tramitar todos los trabajos que no den estricto cumplimiento al Reglamento de Publicaciones y a las Instrucciones de los Autores que se editan en cada número de la Revista. 